\title{
RELAÇÃO ENTRE INDICADORES ANTROPOMÉTRICOS E HEMATOLÓGICOS DE PAIS E SEUS FILHOS
}

\author{
RELATIONSHIP BETWEEN ANTHROPOMETRIC \\ AND HEMATOLOGICAL INDICATORS OF \\ PARENTS AND THEIR CHILDREN
}

\begin{abstract}
Silvana Silveira Soares', Cristiane Fernanda da Silva², Deise Graziela Kern², Ana Paula Sehn², Letícia Welser ${ }^{2}$, Hildegard Hedwig Pohl ${ }^{3}$, Miria Suzana Burgos ${ }^{4}$
\end{abstract}

\section{RESUMO}

Objetivo: Verificar possível associação entre o perfil hematológico e o estado nutricional de trabalhadores e seus filhos. Metodologia: Estudo transversal composto por 25 pais e seus respectivos filhos (25). Avaliou-se índice de massa corporal (IMC), circunferência da cintura (CC), circunferência do quadril $(\mathrm{CQ})$ e relação cintura-quadril (RCQ). Para os indicadores hematológicos, avaliou-se a hemoglobina e o hematócrito. Resultados: 13 pais e 6 filhos apresentam sobrepeso/obesidade e 9 pais e 3 filhos apresentam CC elevada. Entre os filhos, 11 apresentam hemoglobina aumentada e 7 hematócrito aumentado. Não foi observada relação entre os indicadores antropométricos e hematológicos de pais e seus filhos. Com relação aos pais, observou-se relação significativa entre RCQ com hemoglobina $(p=0,002)$ e hematócrito $(p=0,020)$. Entre os filhos, a CC associou-se com a hemoglobina $(p=0,046)$ e com o hematócrito $(p=0,016)$. Conclusão: Não foi encontrada relação dos indicadores antropométricos e hematológicos entre pais e filhos. Por outro lado, os indicadores antropométricos e hematológicos mostraram associação nos pais e nos filhos .

Descritores: Anemia; Obesidade; Adulto; Criança; Adolescente.

\begin{abstract}
Objective: To verify the possible association between hematological profile and nutritional status of workers and their children. Methodology: Cross-sectional study composed of 25 parents and their respective children (25). Body mass index (BMI), waist circumference (WC), hip circumference (HR) and waist-hip ratio (WHR) were evaluated. For hematological indicators, hemoglobin and hematocrit were evaluated. Results: 13 fathers and 6 children were overweight/obese and 9 fathers and 3 children had elevated WC. Among children, 11 have increased hemoglobin and 7 increased hematocrit. There was no relation between the anthropometric and hematological indicators of parents and their children. Regarding the parents, there was a significant relationship between WHR with hemoglobin $(p=0.002)$ and hematocrit $(p=0.020)$. Among children, CC was associated with hemoglobin $(p=$ $0.046)$ and hematocrit $(p=0.016)$. Conclusion: No correlation was found between anthropometric and hematological indicators between parents and children.
\end{abstract}

Descriptors: Anemia; Obesity; Adult; Child; Adolescent.
${ }^{1}$ Acadêmica de Farmácia pela Universidade de Santa Cruz do Sul (UNISC), Santa Cruz do Sul, $\mathrm{RS}$, Brasil.

${ }^{2}$ Graduada em Educação Física pela Universidade de Santa Cruz do Sul (UNISC), Santa Cruz do Sul, $\mathrm{RS}$, Brasil.

${ }^{3}$ Doutora em Desenvolvimento Regional pela Universidade de Santa Cruz do Sul (UNISC), Santa Cruz do Sul, RS, Brasil.

${ }^{4}$ Doutora em Ciências da Motricidade Humana pela Universidade Técnica de Lisboa (UTL), Lisboa, POR, Portugal. 


\section{Introdução}

A anemia, apesar de afetar sistematicamente todo o organismo, pode se manifestar sem ser devidamente diagnosticada, com manifestações clínicas como palidez, fadiga, fraqueza e dificuldade de concentração, as quais são vinculadas ao transporte deficiente de oxigênio para os tecidos alvos. Esta condição pode ser confirmada laboratorialmente, em que alterações hematológicas e bioquímicas, como nos níveis de concentração de Hemoglobina $(\mathrm{Hb})$ e Hematócrito (Hct), apontam níveis menores de eritrócitos do que aqueles ideais de acordo com idade, sexo e outros variantes ${ }^{1-2}$.

A obesidade, por sua vez, trata-se de um acúmulo de gordura corporal muito acima do índice de massa magra, a qual pode aumentar o risco de desenvolvimento de diversas patologias, tais como diabetes mellitus, hipertensão arterial, cardiopatias, dificuldades respiratórias, além de outras complicações. Pode ter origem genética, mas é causada principalmente por uma dieta excessivamente calórica associada a um baixo gasto de energia, criando assim reservas energéticas no tecido adiposo, as quais podem afetar a vida do indivíduo em âmbitos físicos, psicológicos, sociais e ambientais, influenciando diretamente em sua qualidade de vida ${ }^{3}$.

A prevalência de sub e hipernutrição vêm sofrendo alterações em escala mundial, ao que muitos cientistas se referem por "transição nutricional". Enquanto índices de subnutrição vêm decaindo em muitos lugares, nas últimas décadas, os índices de presença de sobrepeso/obesidade na população vêm em uma crescente, principalmente em países em desenvolvimento 4 . Há ainda, a coexistência de ambos estados nutricionais em uma única pessoa, resultado de uma dieta ricamente energética e pobre em micro e macro nutrientes, geralmente encontrada em pessoas carentes ${ }^{5}$.

Apesar de não haver consenso entre os pesquisadores, de quais medidas antropométricas seriam positivamente associadas a indicadores hematológicos, há a certeza da importância da avaliação hematológica, junto à presença de obesidade, considerando que ambos influenciam no desenvolvimento de fatores de risco cardiometabólicos, tais como hipertensão e resistência à insulina-7.

Assim sendo, o presente estudo objetiva verificar possível associação entre o perfil hematológico e o estado nutricional de trabalhadores e seus filhos.

\section{Metodologia}

Foram avaliados, neste estudo transversal, 25 pais, trabalhadores rurais da agroindústria, e seus respectivos filhos, de ambos os sexos e residentes no mesmo domícilio (25). O presente estudo faz parte de uma pesquisa mais ampla, denominada "Novas abordagens em biodinâmica humana para diagnóstico de obesidade, fatores de risco cardiometabólico e diferenciação hematológica em trabalhadores e escolares", aprovado pelo Comitê de Ética em Pesquisa com Seres Humanos da Universidade de Santa Cruz do Sul, sob protocolo número 703.934 e número de CAAE: 26791414.0.0000.5343. O estudo obedeceu aos preceitos éticos legais, conforme direcionamento da comissão nacional de ética na pesquisa (CONEP), em acordo com a resolução n¹96/96, que estabelece as diretrizes para condução de pesquisas com seres humanos. Todos os participantes assinaram o Termo de Consentimento Livre e Esclarecido (TCLE), aceitando a participação no estudo.

Para a avaliação antropométrica foram utilizados os seguintes parâmetros: índice de massa corporal (IMC), circunferência da cintura $(C C)$, circunferência do quadril $(C Q)$ e relação cintura-quadril (RCQ). $O I M C$ foi obtido através das medidas de peso e estatura. Para os filhos, utilizou-se a classificação do CDC/NCHS ${ }^{8}$. Para os pais, os dados foram classificados de acordo com os pontos de corte da Organização Mundial da Saúde ${ }^{9}$. ACC foi avaliada por meio de fita métrica inelástica, considerando como referência a parte mais estreita do tronco entre as costelas e a crista ilíaca. Foram utilizados os critérios de Taylor et al. ${ }^{10}$, para classificação dos dados dos filhos, considerando CC elevada o percentil $>80$. Para os pais, foram usadas as recomendações de Heyward ${ }^{11}$, em que CC maior que $100 \mathrm{~cm}$ em homens e $88 \mathrm{~cm}$ em mulheres já é considerada como obesidade e fator de risco para cardiopatias e doenças metabólicas. Para a $C Q$, considerou-se o nível do trocânter maior, como referência. A RCQ foi calculada através da divisão dos valores de CC pela CQ.

Os níveis de hemoglobina e o hematócrito foram utilizados como parâmetros hematológicos. Após a coleta, as amostras de sangue foram armazenadas em tubos contendo EDTA, mantidos em homogeneizador até o momento da análise, a qual foi realizada em equipamento automatizado Sysmex XS800i. Os dados obtidos foram classificados de acordo com os critérios estabelecidos pela Organização Mundial da Saúde ${ }^{12}$. 
A análise estatística dos dados foi realizada através do software SPSS v. 20.0 (IBM, Armonk, NY, EUA). Foi utilizada a estatística descritiva, através de frequência e percentual, além dos valores médios e desvio-padrão. Para testar a associação entre os valores contínuos dos indicadores antropométricos e hematológicos, foi utilizado o teste de correlação de Spearman.

\section{Resultados}

A tabela 1 apresenta as características descritivas dos sujeitos. Observa-se, para os indicadores antropométricos, que 13 pais e 6 filhos apresentam excesso de peso (sobrepeso/obesidade) para o IMC e 9 pais e 3 filhos apresentam CC elevada. Com relação aos indicadores hematológicos, as alterações foram mais relevantes entre os filhos, sendo que 11 apresentam hemoglobina aumentada e 7, hematócrito aumentado.

Tabela 1 - Características descritivas dos sujeitos com relação aos indicadores antropométricos e hematológicos

\begin{tabular}{c|c|c}
\hline \multirow{2}{*}{} & Pais (N=25) & Filhos (N=25) \\
\cline { 2 - 3 } & $\mathbf{n \%}$ & $\mathbf{n \%}$ \\
\hline Idade* & $37,5(6,6)$ & $12,0(3,1)$ \\
\hline Sexo & $6(24,0)$ & $15(60,0)$ \\
\hline Masculino & $19(76,0)$ & $10(40,0)$ \\
\hline Feminino & \multicolumn{2}{|c}{}
\end{tabular}

\begin{tabular}{c|c|c}
\hline \multicolumn{3}{l}{ Indicadores antropométricos } \\
\hline IMC $\left(\mathrm{kg} / \mathrm{m}^{2}\right)^{*}$ & $26,20(4,99)$ & $19,89(3,97)$ \\
\hline Normal & $12(48,0)$ & $19(76,0)$ \\
\hline Sobrepeso & $8(32,0)$ & $3(12,0)$ \\
\hline Obesidade & $5(20,0)$ & $3(12,0)$ \\
\hline CC $(\mathrm{cm})^{*}$ & $80,92(11,28)$ & $65,49(9,14)$ \\
\hline Normal & $16(64,0)$ & $22(88,0)$ \\
\hline Elevada & $9(36,0)$ & $81,98(12,76)$ \\
\hline CQ (cm)* & $101,29(10,41)$ & $0,79(0,05)$ \\
\hline RCQ* & $0,80(0,06)$ &
\end{tabular}

\begin{tabular}{c|c|c}
\hline \multicolumn{3}{l}{ Indicadores hematológicos } \\
\hline Hemoglobina $(\mathrm{g} / \mathrm{dL})^{*}$ & $13,53(1,43)$ & $13,39(1,07)$ \\
\hline Normal & $25(100,0)$ & $14(56,0)$ \\
\hline Aumentada & $0(0,0)$ & $11(44,0)$ \\
\hline Hematócrito $(\%)^{*}$ & $40,51(3,96)$ & $39,24(2,78)$ \\
\hline Baixo & $4(16,0)$ & $0(0,0)$ \\
\hline Normal & $21(84,0)$ & $18(72,0)$ \\
\hline Aumentado & $0(0,0)$ & $7(28,0)$ \\
\hline
\end{tabular}

*Dados expressos em média (desvio-padrão). IMC: índice de massa corporal;

CC: circunferência da cintura; CQ: circunferência do quadril; RCQ: relação cintura-quadril.

Na comparação dos indicadores antropométricos e hematológicos de pais e seus filhos, não foi observada relação (Tabela 2). 
Tabela 2 - Correlação dos indicadores antropométricos e hematológicos entre pais e filhos

\begin{tabular}{c|c|c}
\hline & Correlação* & p \\
\hline \multicolumn{2}{|c}{ Indicadores antropométricos } \\
\hline IMC & 0,307 & 0,135 \\
\hline CC & 0,152 & 0,467 \\
\hline CQ & 0,334 & 0,102 \\
\hline RCQ & 0,115 & 0,584 \\
\hline
\end{tabular}

Indicadores hematológicos

\begin{tabular}{c|c|c}
\hline Hemoglobina & 0,303 & 0,142 \\
\hline Hematócrito & 0,318 & 0,122 \\
\hline
\end{tabular}

IMC: índice de massa corporal; CC: circunferência da cintura; CQ: circunferência do quadril; RCQ: relação cintura-quadril; * correlação de Spearman; diferenças significativas para p<0,05.

Quando comparados os indicadores antropométricos com os indicadores hematológicos, observou-se relação significativa entre RCQ com hemoglobina $(p=0,002)$ e hematócrito $(p=0,020)$ dos pais. Entre os filhos, a CC associou-se com a hemoglobina $(p=0,046)$ e com o hematócrito $(p=0,016)$. Ambos os dados estão descritos na tabela 3.

Tabela 3 - Correlação entre indicadores antropométricos e hematológicos, estratificado por pais e filhos

\begin{tabular}{c|c|c|c|c}
\hline \multirow{2}{*}{} & \multicolumn{2}{c|}{ Hemoglobina } & \multicolumn{2}{c}{ Hematócrito } \\
\cline { 2 - 5 } Pais & Correlação* & $\mathbf{p}$ & Correlação* & p \\
\hline IMC & 0,267 & 0,197 & 0,201 & 0,334 \\
\hline CC & 0,351 & 0,085 & 0,263 & 0,204 \\
\hline CQ & 0,132 & 0,530 & 0,119 & 0,572 \\
\hline RCQ & 0,589 & 0,002 & 0,461 & 0,020 \\
\hline
\end{tabular}

\section{Filhos}

\begin{tabular}{c|l|l|l|l}
\hline IMC & 0,323 & 0,115 & 0,370 & 0,069 \\
\hline CC & 0,402 & 0,046 & 0,476 & 0,016 \\
\hline CQ & 0,295 & 0,153 & 0,358 & 0,079 \\
\hline RCQ & 0,025 & 0,906 & 0,035 & 0,868 \\
\hline
\end{tabular}

IMC: índice de massa corporal; CC: circunferência da cintura; CQ: circunferência do quadril; RCQ: relação cintura-quadril; *correlação de Spearman; diferenças significativas para $p<0,05$.

\section{Discussão}

Ao realizar busca na literatura nacional e internacional, foram constatados escassos estudos, avaliando as relações de perfis hematológico e antropométrico entre pais e filhos. Não foi observada relação na comparação dos indicadores antropométricos e hematológicos de pais e seus filhos no presente estudo. Em estudo, avaliando a transição nutricional brasileira, autores encontraram associação significativa entre o peso das mães e o dos filhos, sugerindo, ainda, um aumento drástico de IMC na passagem de uma geração para outra, principalmente em países que passam pela chamada "transição nutricional", como é o caso do Brasil'5. 
Dos 25 trabalhadores no presente estudo, 13 apresentaram excesso de peso (sobrepeso/obesidade), para o IMC, e 9 CC elevada. Em uma análise do estado nutricional nacional baseado em censos realizados pelo Instituto Brasileiro de Geografia e Estatística (IBGE), um estudo encontrou que 1 em cada 2 brasileiros adultos possuía sobrepeso e 1 em cada 7 possuía obesidade, entre 2008 e 2009, sendo que a projeção de aumento dessa população era de 4,7\% para homens e $2,5 \%$ para mulheres, a cada ano ${ }^{5}$.

Entre os 25 filhos avaliados neste estudo, 24\% apresentam excesso de peso (sobrepeso/obesidade) para o IMC. Estudo realizado no estado do Tocantins encontrou $15 \%$ de prevalência de sobrepeso/obesidade em crianças de idade escolar conforme IMC ${ }^{13}$.

Dos parâmetros antropométricos analisados no presente estudo, encontrou-se relação significativa positiva somente entre RCQ com hemoglobina e hematócrito dos pais. Autores já haviam encontrado relação, porém negativa, de IMC e hemoglobina em sul-africanos; entretanto, encontrando relação positiva entre CC e hemoglobina ${ }^{14}$. Outros autores não encontraram relação da CC e do IMC com parâmetros hematológicos em italianos, mas após um ajuste com as variáveis sexo, idade e o hábito de fumar, encontraram uma relação positiva entre eles, o que foi atribuído à gordura abdominal e marcadores de resistência à insulina ${ }^{6}$. Um estudo encontrou uma relação positiva entre CC e hemácias, hemoglobina e hematócrito em americanos voluntários do US National Health and Nutrition Examination Survey (NHANES) ${ }^{15}$. Outro, encontrou associação entre hemácias, hematócrito e hemoglobina e índices de IMC e RQC em adultos chineses ${ }^{16}$. Um estudo relatou que mulheres adultas tinham maior risco de síndrome metabólica, quanto maiores seus níveis de hemoglobina, hematócrito, plaquetas e leucócitos, embora hemoglobina e hematócrito, parâmetros aqui avaliados, não tenham se associado por si só com os parâmetros antropométricos avaliados por eles ${ }^{17}$.

Entre os filhos, a CC associou-se com hemoglobina e com hematócrito em nossa pesquisa, assim como relata também um estudo com adolescentes pernambucanos, que encontrou associação entre hemácias e hemoglobina com $\mathrm{CC}^{18}$. Diferentemente, autores que avaliaram escolares mexicanos não encontraram associação entre hemoglobina $\mathrm{e}$ IMC ${ }^{19}$. Na Turquia, autores demonstraram um aumento de hematócrito em obesos mórbidos em relação a adolescentes eutróficos, pelo IMC, apesar de não ter encontrado o mesmo, em relação a hemoglobina em crianças e adolescentes ${ }^{20}$.

Além disso, não foi encontrado, em nosso estudo, nenhum indivíduo que apresentasse, em conjunto, excesso de peso e anemia, estando, portanto, abaixo do percentual brasileiro (1,2\% entre as crianças e $13,6 \%$ das mulheres), como estimado por estudo anterior ${ }^{21}$.

Apesar da relevância do presente estudo, apresentamos algumas limitações, como o tamanho amostral pequeno e o delineamento do estudo, o qual não pode estabelecer relação de causa e efeito. Além disso, a amostra dos filhos é composta por crianças e adolescentes, não sendo avaliado o estágio maturacional. Contudo, o estudo aborda pontos positivos relevantes, pois apresenta dados hematológicos de pais e seus filhos, aspecto que dificilmente é abordado nas pesquisas que exploram esta relação.

\section{Considerações Finais}

Não foi encontrada relação dos indicadores antropométricos e hematológicos entre pais e filhos. Entre os pais, a $\mathrm{RCQ}$ associou-se com hemoglobina e hematócrito. A CC apresentou relação com hemoglobina e hematócrito entre os filhos. Sugerem-se novos estudos, com uma amostra maior, para confirmar os achados da presente avaliação.

\section{Referências}

1. Camaschella, C. Iron-deficiency anemia. N Engl J Med. 2015; 373:484-486.

2. De Andrade Cairo RC, Rodrigues Silva L, Carneiro Bustani N, Ferreira Marques CD. Iron deficiency anemia in adolescents; a literature review. Nutr Hosp. 2014;29(6):1240-9.

3. Pimenta FBC, Bertrand E, Mograbi DC, Shinohara H, Landeira-Fernandez J. The relationship between obesity and quality of life in Brazilian adults. Frontiers in Psychology, Lausanne, v. 6, 2015.

4. Popkin BM. Contemporary nutritional transition: determinants of diet and its impact on body composition. Proc Nutr Soc. 2011;70(1):82-91.

5. Conde WL, Monteiro CA. Nutrition transition and double burden of undernutrition and excess of weight in Brazil. Am J Clin Nutr. 2014;100(6):1617S-22S. 
6. Barazzoni R, Cappellari GG, Semolic A, Chendi E, lus M, Situlin R, et al. The Association between Hematological Parameters and Insulin Resistance is modified by Body Mass Index - Results from the North-East Italy MoMa Population Study. PLoS One. 2014; 9(7): e101590.

7. Altunoğlu E, Müderrisoğlu C, Erdenen F, Ulgen E, Ar MC. The impact of obesity and insulin resistance on iron and red blood cell parameters: a single center, cross-sectional study. Turk J Haematol. 2014;31(1):61-7.

8. Centers for Disease Control and Prevention/National Center for Health Statistics (CDC/NCHS). CDC Growth Charts: United States. 2000. Disponível em: <http://www.cdc.gov/growthcharts>. Acesso em: 20 out. 2016.

9. World Health Organization (WHO). Obesity: preventing and managing the global epidemic. Report of a World Health Organization Consultation. Geneva: World Health Organization. WHO Obesity Technical Report Serie;, 2000. n. 284, 256 p. 10. Taylor RW, Jones IE, Williams SM, Goulding A. Evaluation of waist circumference, waist-to-hip ratio, and the conicity index as screening tools for high trunk fat mass, as measured by dual-energy X-ray absorptiometry, in children aged 3-19 y. Am J Clin Nutr. 2000 Aug;72(2):490-5.

11. Heyward VH. Avaliação física e prescrição de exercício: técnicas avançadas. 4ª . ed. Porto Alegre: Artmed; 2004.

12. World Health Organization (WHO). Iron deficiency anaemia: assessment, prevention and control. A guide for programme managers. Geneva: WHO/UNICEF/UN; 2001.

13. Rezende AAB, Rodrigues ESR, Alves GPLAG, Alves MPA, Reis NM, Moreira RDF. Fatores de risco cardiovascular em crianças de uma escola da rede pública do município de Gurupi-TO. Amazônia: Sci Health, 2014; 2(4): 2-8.

14. Choma SS, Alberts M, Modjadji SE. Conflicting effects of BMI and waist circumference on iron status. J Trace Elem Med Biol. 2015;32:73-8.

15. Vuong J, Qiu Y, La M, Clarke G, Swinkels DW, Cembrowski G. Reference intervals of complete blood count constituents are highly correlated to waist circumference: Should obese patients have their own "normal" values? Am J Hematol. 2014;89(7):671-7.

16. Jiang Y, Zeng J, Chen B. Hemoglobin combined with triglyceride and ferritin in predicting non-alcoholic fatty liver. J Gastroenterol Hepatol. 2014;29(7):1508-14.

17. Lohsoonthorn V, Jiamjarasrungsi W, Williams MA. Association of hematological parameters with clustered components of metabolic syndrome among professional and office workers in Bangkok, Thailand. Diabetes Metab Synd: Clin Res Rev. 2007; 1(3): 143-149.

18. Ferreira LC, Da Silva HJ, Lins TA, Do Prado WL. Relationship between lipid and hematological profiles with adiposity in obese adolescents. Rev Bras Hematol Hemoter. 2013;35(3):163-6.

19. Alcocer-Aguilar JL, Hernández-Escalante VM, Marín-Cárdenas AD, Castro-Sansores C, Cabrera-Araujo Z. Ingestión baja de hierro y anemia en escolares con sobrepeso y obesidade, Ciencia Humanismo Salud. 2015;2(1):4-9.

20. Donma O, Donma MM, Nalbantoglu B, Topcu B, Tulubas F, Aydin F, et al. The Importance of Erythrocyte Parameters in Obese Children. International Scholarly and Scientific Research \& Innovation. 2015;9(5):337-340.

21. Rivera JA, Pedraza LS, Martorell R, Gil A. Introduction to the double burden of undernutrition and excess weight in Latin America. Am J Clin Nutr. 2014 Dec;100(6):1613S-6S. 


\section{Silvana Silveira Soares}

Endereço para correspondência - Av. Independência, $n^{\circ} 2293$ (Departamento de Educação Física e Saúde), Bairro: Universitário, CEP: 96815900 , Santa Cruz do Sul, RS, Brasil.

E-mail: silvanasilveiras@hotmail.com Lattes: http://lattes.cnpq.br/127071415413347

Cristiane Fernanda da Silva - cris.cfs@hotmail.com

Deise Graziela Kern - deisekern@hotmail.com Ana Paula Sehn - ana_psehn@hotmail.com Letícia Welser - leticia.welser@bol.com.br Hildegard Hedwig Pohl - hpohl@unisc.br Miria Suzana Burgos-mburgos@unisc.br

\section{Enviado em 23 de março de 2017. Aceito em 07 de junho de 2017.}

